\title{
Evaluation of a New Multipurpose Centrifugal Mechanism for Nut Process
}

\begin{abstract}
Post processing, processing and transportation of pistachio, have great effects on its quality, food value, physical properties and marketability of this product. Then any applicable research for processing of pistachio, have direct and indirect effects on developing technology of this industry and as a result, farmers can get more income. In this study, various methods of pistachio and other nuts processing in recent years have been investigated and after that, new suitable and the multipurpose centrifugal mechanism for nuts (especially for pistachio) processing has been presented. The mechanism is able to do hulling, washing, draining and feeding out for sorting purposes, all in one. The machine has been constructed in the laboratory and loaded by pistachio in two treatments and by walnuts in four treatments. In both hulling and draining processes good results obtained. Time did not play as an important role for hulling process. Bigger nuts with higher inertia like walnuts were hulled better in this system. Some of the advantages of this new machine are simple mechanisms, avoiding from using any complex part, capable of automation, simple usage, simple maintenance and finally low cost to construct.
\end{abstract}

Keywords: Processing of Pistachio, Processing of Nut; Hulling and Draining, Multipurpose Machine.

\section{Introduction}

Ever-increasing pistachio usage, high food value, production- exportation growth and Iran's portion of world pistachio production, necessitated more investigation and attention to this product. Mechanized process of pistachio before and after the harvesting has great impacts on quality, food value, competitive market and the world production of this strategic product. Considering the 427097 tonnes per year 2012 average production of pistachio in Iran, which includes almost $43.7 \%$ of the world production (FAO, 2014), highlights the significance of mechanization of the pistachio process in Iran. Therefore, any applicable investigation for the better production process, directly or indirectly, has pronounced effects on farmer's income.

Different methods of pistachio process have been proposed by corresponding large companies. Pistachio process algorithm in one of the largest centers of this product processing, in California (VincentCorp, 2007), is discussed here as a good example. Throughout this process, the green hull is removed, which is performed by so rough abrasives with water. Thereafter, the product is transferred directly to the water tank, so floating pistachio nuts which have low quality separate from the sinked high quality pistachio nuts. The next step is to dry the product and finally it is sorted based on color, size, etc. packed and transported to the market. Based on the researches conducted by the specialists, removing the hull from 
pistachio requires more energy consumption compared to other processes such as washing and draining step. Consequently, comparisons of different pistachio hullers have been made and studied throughout these articles (Jay \& Bertram, 1977; Joseph \& Benjamin, 1981, 1993; Mahmoodi, Mohammdi, Jafari, \& Fathollahzadeh, 2007; VanmarkCorp, 2004).

Pistachio washing is performed after or simultaneously with the hulling process, while in newly made machines, some injectors are provided to spray water on pistachio (Jay \& Bertram, 1977). While water spraying, performs the washing, it increases the efficiency of the hullers remarkably (VanmarkCorp, 2004).

Based on the researches performed by some scientists, drying pistachio immediately after the washing process has great effects on reduction of fungous diseases growth, such as Aflatoxin (Hyang, Hyun, \& Hyun, 2006). Drying machine constructed by Nakhaeinezhad (Nakhaeinezhad, 2002), includes an oscillating grain and a warm air blower, which perform the drying process. A kind of precise dryer was constructed by Kashaninejad et al (2005) which was equipped with some temperature, moisture and blown air pressure sensors which were able to measure those parameters online (Kashaninejad, Mortazavi, Safekordi, \& Tabil, 2005; Kashaninejad \& Tabil, 2004).

A new mechanism assembled on a chassis equipped with power transmission and electromotors that called multipurpose machine is presented in this study. First, it is briefly described how the machine works. Second, the evaluation process is explained. Afterward, measured data plotted against proper variables are presented and discussed to reach final conclusions.

\section{Machine Description}

A general view of the machine is shown in FIG. 1. Modeling and mechanical analysis of this machine is performed using Catia V5 engineering software which would enable us to analyze the components and do the optimization. The major components which the machine includes are the frame, electromotors, control panel, power transmission systems, Outside Abrasive Cage (OAC), Inside Abrasive Cage (IAC), curved bottom, air blower lid, collecting waste hull/water chamber and quick discharge gate. It's worth nothing but has to be indicated that, in the first try of construction, the collecting waste hull/water chamber is not assembled on the machine. It is for having better visualization in the evaluation process.

As seen in the FIG. 1, the restricted space between the outside and inside abrasive cages and the bottom constitutes the mechanism as a process chamber of the machine. The nut is poured into the process chamber and then, hulling, washing, draining and discharging processes are performed respectively. The required power for different processes is provided by two $220 \mathrm{~V}$ electromotors with maximum power and 
speed of $2.2 \mathrm{~kW}$ and $900 \mathrm{RPM}$, respectively. As shown in FIG. 1, the OAC is rotated by the electromotor 1 while IAC and the curved bottom are rotated by the electromotor 2.

Controlling the speed and direction of rotation via the control panel helps performing the processes. Control panel, includes inverters, contactors, stop and start switches and chronometers.

After software designation and optimization of each part, a laboratory sample of the multipurpose machine is constructed as presented in FIG. 2. In the construction process, the hardest and time consuming section was handling the process chamber (FIG. 2b). It was modified three times to reach a proper performance. For each motor, the control panel contains a frequency inverter which would able us to change the rotational speed of the cages in a continual way. Programmable inverters helped us to have an automated and regular multi-process. The reader is referred to (Mahmoodi, Khazabi-Khasraghi \& Jafari, 2007) where details of the design, construction and modification processes as well as main dimensions are included in.

\subsection{Hulling operation}

For hulling operation, pistachio will be poured into the process chamber. Now there are three modes. The first one is the clockwise rotation of the OAC, the second one is the counterclockwise rotation of the IAC and the bottom and the third mode is the simultaneously opposite rotation of the former and the latter which each has a portion in performing the hull removing step (FIG. 1). As you know, different species and sizes of pistachio nuts have various strengths against the mechanical damages. So modification of the hulling aggressiveness as it was done during construction, can improve the efficiency of this step significantly. Rotation of the OAC, IAC or their opposite simultaneous rotations and alternation of the speed of the electromotors, can provide different pistachio hull removing aggressivenesses. It seems clear that when these two cages rotate oppositely, hull removing process occurs with high capacity and performance. Therefore, in this study the machine follows this rule in all tests and the rotational direction of cages has been set up in the opposite direction in every test. One of the efficient parameters on performance of pistachio hull removing machines and other similar ones is the agitation. Therefore, the machine bottom has a curved design which rotates by the inside cage simultaneously. This curvature causes the agitation. This method is used widespread in different machines such as washing machines. The bottom curvature, during the rotation, more than agitating the pistachio helps abrasion of the green hull due to the contact with the vertical abrasive walls (FIG. 3a and 3b) which improve the efficiency and machine capacity. The way the green hull is removed is shown in FIG. 3a. Inside/outside abrasive cages and curved bottom, have $4 \mathrm{~mm}$ distance slots (see FIG. 2b) which perform like the kitchen grates. Cutting edges of the slots in contact with the pistachio nuts, remove the green hulls partially. The forces exerted 
on the pistachio nut which helps it stand against the wall slots include the internal friction among the nuts, friction due to the nut-wall contact and the centrifugal force. Nuts which stick to the OAC because of centrifugal force cannot continue due to confronting with agitation and reversed rotation of IAC.

\subsection{Washing Operation}

The machine has been designed in a way to wash the product immediately after the hull removing step. The shorter transferring time from hulling step to washing process lowers the possibility of cross contamination and spread. In this machine, instantly after hull removing step, electromotor 1 is stopped and the rotation speed of the electromotor 2 is decreased to fulfill the agitation. Water distribution and rotation of the curved bottom cause the washing operation ends soon. Waste materials and removed hull pieces which have not left the pile of the pistachio nuts, go out of the washing container through the slots by water flow.

\subsection{Moisture Draining Operation}

The multipurpose machine presented in this article is able to drain the moisture, but not able to dry. Draining operation follows the washing one. On this machine the draining operation is performed without any need for transferring the nuts out of the machine to another independent draining machine and consequently helps save time and cost. For performing this operation, both of the electromotors rotate in the same direction to prevent the friction between the abrasive cages and nuts. Here the maximum speed of electromotors is used. The flow of air blows through the mass of nuts, which comes from the high rotation speed. Due to the centrifugal force, a portion of the moisture is taken from the porosities among the nuts and the surface of them and consequently goes out through the slots of the OAC. The air flow as shown in FIG. 3 comes vertically to the chamber, absorbs the moisture and finally leaves it in radial direction. If agitation and draining operations are performed simultaneously the efficiency of the machine increases remarkably. This is possible by fast driving both curved bottom and OAC but with a slight difference in rotation speed.

\subsection{Discharging Operation}

A quick discharge way has been considered in this machine. To use this method, electromotor1 is stopped and the rotation speed of the eletromotor 2 is decreased as much as possible. Then the quick discharge gate provided on the OAC (FIG. $1 \mathrm{~b}$ and $2 \mathrm{~b}$ ) is opened, and consequently the processed production is discharged quickly. By controlling the rotary speed of the curved bottom (electromotor 2) the discharging operation would be adjusted which can be used for sorting operation next to the machine. 


\section{Evaluation Methodology}

The machine is evaluated not only for pistachio nuts, but also for walnuts. Hulling process is assessed for both, but draining process is only assessed for pistachio nuts. Described treatments below are suggested by experts in Iranian Pistachio Research Institute (PRI).

\subsection{Treatments for Pistachio Nuts}

Table 1 explains treatments used for assessing hulling operation for pistachio nuts (Persian variety: Akbari). Minus sign of the IAC rotation speed describes the opposite rotational direction of the cage compare to OAC. As seen, two treatments are used. They reveal how rotation speeds of OAC and IAC affect the hulling process. The measured average moisture content of pistachio's green hull is $68.1 \%$ (dry based) for both speed variations.

In each treatment, power consumption of each abrasive cage is measured in every one minute. It is clear that a considerable portion of the power is consumed by internal friction of the machine. It has been assumed to be constant during the evaluation process. So, at the first of each treatment the machine is operated without nuts and the empty consumed power is measured. Then the relevant treatment is applied and the total power consumption is recorded. The empty power is reduced from the total one and the pure power consumption is calculated. Each treatment is repeated four times $(\times 4)$ to reach reliable average results.

For assessing ability for moisture draining of pistachio, four different rotation speeds are used for OAC: $62.8-78.5-86.4-94.2$ all in $\mathrm{Rad} / \mathrm{s}$. To have enough agitations in the draining process, IAC is set at $5.0 \%$ lower speed than that of OAC in each step. This reveals how rotation speeds of the process chamber affect the draining process in this machine.

After hulling operation, the product is washed and five samples are taken to the laboratory for measuring the whole moisture content before draining. Then, $4(\times 4) \mathrm{kg}$ of the washed product is drained under the different rotation speeds of the process chamber for 15 minutes in each test. After draining of each treatment, five samples are taken to the lab to measure the whole moisture content.

\subsection{Treatments for Walnut}

According to designing principle of this machine, nuts with big dimensions, heavy weight and thick green hull must have significant effects on the hulling process. Therefore, as an upstream treatment, it has been decided to assess hulling ability of the machine for peeling walnut as one of the biggest nuts in Iran 
(Persian variety: Kaghazi). Table 2 describes treatments considered for assessing the hulling process. Minus sign of the IAC rotation speed describes the opposite rotational direction of the cage compare to OAC. The measured average moisture content of walnut green hull is $87.6 \%$ (wet based) in all treatments. Each treatment is repeated four times $(\times 4)$ to reach reliable average results.

Power consumption along the time is also measured here. It has been recorded for every minute in treatments $\mathrm{A}$ and $\mathrm{B}$, and for every 30 seconds in treatments $\mathrm{C}$ and $\mathrm{D}$, for each abrasive cage separately. This assessment reveals how time spending and rotation speed of OAC and IAC affects the walnut's hulling process.

\subsection{Statistical Analysis}

The experiments were conducted in four replications for each time and rotation speed of the cages, then the average values reported. The analysis of variance (ANOVA) was carried out on a completely randomized design experiment using SPSS16 software. The one-way ANOVA test was used to determine significant effects of each treatment, and significant differences of means were compared using the Duncan's multiple range test at $5 \%$ significance level.

\section{Results and Discussions}

All treatments mentioned above are applied on the new multipurpose machine presented in this research. The obtained results are separated into two sections: Evaluation for pistachio nuts, and for walnuts.

\subsection{Evaluation for Pistachio Nuts}

Green hulls are peeled and forced out of the process chamber as expected. The output of both treatments is assorted below to three levels of quality which is done by hand and one by one:

- Complete peeled level: This level mentions to nuts, which the green hull is completely peeled. This level introduced as the performance of the machine for hulling pistachio.

- Medium condition level: This level mentions to nuts, which at least half of the surrounded hull is removed.

- Untouched level: Nuts with untouched green hull is placed in this level.

As seen in FIG.4, averaged performance of the machine is measured 57.14\% (tolerance error 3.70\%) in treatment A. This is much better for treatment B, where it is $66.67 \%$ (tolerance error $2.61 \%$ ). In the third level of quality, untouched pistachios is $22.86 \%$ of the total output in treatment A which decreased in 
treatment B up to $2.78 \%$. This exhibits the process chamber has better performance in treatment $\mathrm{B}$. This observation indicates that the increasing rotation speed of OAC has much favorable effect on the performance rather than that of IAC in hulling process. So, OAC is doing much more than IAC in the hull removing process. Seems it's because of centrifugal force which forces the product to the OAC wall. The agitation due to the curved floor of the IAC makes a vertical flow inside the product. It has been seen during tests as well. While the chamber is full of product the agitation is starting to lead the flow from the OAC inner wall to the IAC outer wall. So, IAC here is helping to increase the capacity of the machine in the hull removing process.

The results of variance analysis which was carried out to examine the effect of treatments on hulling performance are shown in Table 3 for both pistachios and walnut. As seen, the effect of the rotation speed of the cages on the hulling performance is significant at the $1 \%$ of the probability level for pistachio nut.

Table 4. shows the results of the mean comparison of hulling performance in different levels of treatments for both pistachios and walnut. It is apparent that either variation in rotation speed of OAC and IAC had a significant effect on the hulling performance of pistachio $(p<0.99)$.

As seen in Table 5., averaged power consumption of OAC and IAC in treatments A is 1.237 and $0.54 \mathrm{~kW}$ respectively, since in treatment B they are 1.017 and $0.66 \mathrm{~kW}$, respectively. In treatment $\mathrm{B}$ has reduced consumed power of OAC for $17.78 \%$. This indicates that treatment B will save more energy than treatment A.

The whole moisture content of the hull removed nuts consists of two un-separable parts: surface water/moisture and internal moisture contents. For the surface water/moisture content, it's clear that a significant increasing and decreasing happens during the washing and draining process, respectively, since for the internal moisture content part, there is no time to get a big change in both processes. The results of the draining evaluation are briefed in FIG.6. As expected, the whole moisture content is decreased while the rotation speed of the process chamber is increased. A linear relation ( $\mathrm{Y}=0.1446 \times \mathrm{X}+$ 5.4062) with $R^{2}=0.95$ is observed, where $Y$ is the dry based whole moisture reduction in percentage and $\mathrm{X}$ is the rotation speed of the process chamber in radian per second. This indicates that increasing rotary speed by $10 \mathrm{rad} / \mathrm{s}$ causes to reduction the whole moisture by about $1.4 \%$, which a significant part of the reduction must be related to the surface water/moisture content.

\subsection{Evaluation for Walnut}

The outputs of each treatment are assorted to five levels. Educated experts helped us to accomplish this step. This is done by hand and one by one. The horizontal axis of the chart illustrated in FIG.7 introduced 
as hulling quality which is the percentage of the peeled hull of each nut. A level of $100 \%$ is introduced as performance of the machine. As seen, the performance of the machine for hulling bigger nuts are also acceptable. Comparing treatments $A \& B$ to $C \& D$ clearly indicates that doubling the processing time has insignificant increase about $4.5 \%$ for performance.

Satisfying results are obviously observed in treatment B. The whole product is completely hulled. The next rank belongs to treatment (D) with about $4.5 \%$ lower performance. Same as pistachio nuts, rotation speed of OAC here also has a significant effect on the hulling performance.

As it can be found from Table 3, the effects of hull removing time and rotation speed of the cages on the hulling performance are significant at the $1 \%$ of the probability level for walnut. From a mean comparison of hulling performance (Table 4) it is found that there is no significant difference between treatments $\mathrm{A}$ and $\mathrm{C}$ which means the treatment of time has no significant effects on the hulling performance of the machine ( $p<95)$. It's also found that there is no significant interaction between hull removing time and rotation speed of the cages at the $5 \%$ probability level.

As seen in Table 6, increasing the rotation speed of OAC has insignificant increase in the power consumption in the hulling process. Same as pistachio process, the power consumed by OAC is almost double of IAC here. Better distribution of power consumption in treatment B is also observed here. Like the evaluation for pistachio, the OAC consumes power almost double of the IAC here too (power flow in FIG. 8). In treatments $A$ and $B$, it's seen that power flows start with a high consumption value (OAC(A): $1.4 \mathrm{~kW}, \operatorname{IAC}(\mathrm{A}): 1 \mathrm{~kW}, \mathrm{OAC}(\mathrm{B}): 1.5 \mathrm{~kW}$ and $\operatorname{IAC}(\mathrm{B}): 0.75 \mathrm{~kW})$, continue in almost a constant value and end with an insignificant smooth reduction $(\mathrm{OAC}(\mathrm{A}): 1.1 \mathrm{~kW}, \mathrm{IAC}(\mathrm{A}): 0.78 \mathrm{~kW}, \mathrm{OAC}(\mathrm{B}): 1.02 \mathrm{~kW}$ and IAC(B): $0.85 \mathrm{~kW}$ ). This exhibits that same as pistachio process the power consumption does not grow up linearly with the capacity. This is a normal response of the centrifugal mechanism while starts, continues and ends a normal hulling process.

\section{Conclusions}

A centrifugal mechanism is used and a multipurpose process chamber is designed and constructed to offer an all in one nut processing machine for farmers. Suitable safety, the simple mechanism of belt and pulley for the power transmission, easy and simple assemblage, the capability of being equipped with automatic systems and capable of doing several processing works via one machine were the main purposes for designing and constructing this machine. Evaluation of the machine exhibited that the performance of the process chamber for pistachio nuts and also walnuts in hulling step is acceptable. It was also found that outer abrasive cage (OAC) has a more significant effect on the hulling process than inner abrasive cage 
(IAC). Time is not the key to reach a high efficiency hulling process. This is also confirmed by the analysis of variance. Doubling the process time increased the hulling performance just to $4.5 \%$. Jumping to bigger nuts like walnut increased the hulling performance. More inertia of walnut seems to be the reason of this increment. It's also found that the power consumption of the machine in the hulling process did not grow up linearly with the capacity. The acceptable draining ability of this machine was confirmed in this study as well. More rotation speed of the process chamber absolutely increased the draining ability of the machine. According to the results, it's found that a calibrated version of this mechanism can be used for hulling other nuts as well. For a laboratory sample, satisfying results approved that there is enough potential toward a commercial version of this multipurpose machine. Being multipurpose and being possible to own by small scale farmers and features discussed above were the main challenges of the authors to design this machine. The capacity is sacrificed to get the new features here and with the current situation the capacity alone is not marketable. Maybe in the commercial version this problem gets resolved via adding some new options. By the way, if we consider this weakness beside the new features gained in this research, the whole machine seems to be marketable.

\section{Acknowledgements}

We are very thanks full of Iran National Science foundation (INSF) and Agricultural Engineering Research Institute (AERI) for funding this research and giving us scientific consultation.

\section{References}

FAO. (2014). Statistics of Quantity of Pistachio Production in Iran.

Hyang, S. C., Hyun, J. K., \& Hyun, E. O. (2006). Determination of Aflatoxin 236 levels in nuts and their products consumed in South Korea. Food Chemistry, 102, 385-391.

Jay, J. M., \& Bertram, E. S. (1977). Pistachio Huller. P N: US 4034665 A.

Joseph, V., \& Benjamin, V. (1981). Method of hulling pistachio nuts. P N: US 4353931 A.

Joseph, V., \& Benjamin, V. (1993). Pistachio Nuts Hulling Apparatus. P N: US 5245918 A.

Kashaninejad, M., \& Tabil, L. G. (2004). Drying characteristics of purslane (Portulaca oleraceae L.). Drying Technology, 2(9), 2183-2200.

Kashaninejad, M., Mortazavi, A., Safekordi, A., \& Tabil, L. G. (2005). Thin-layer drying characteristics and modeling of pistachio nuts. Food Engineering, 78, 98-108.

Mahmoodi, E., Khazabi-Khasraghi, K., \& Jafari, A. (2007). Kinetic Analysis, Power Calculation and Safety Factor Determination of a Two-Corrosive-Cage Pistachio Peeler. Iranian Food Science and Technology Research, 3(1), 37-44. 
Mahmoodi, E., Mohammdi, A., Jafari, A., \& Fathollahzadeh, H. (2007). Performance Analysis and Comparison of Different Pistachio Peelers. Paper presented at the The First Congress of Processing and Packaging Pistachio in Iran, Mashhad. Iran.

Nakhaeinezhad, M. (2002). Pistachio Pre- Dryer. P N: US 6499228 B2.

VanmarkCorp. (2004). Peeler, Scrubber and Washer series 2800. Equipment For The Food Industry.

VincentCorp. (2007). California Pistachio Processing Plant Flow Chart. 


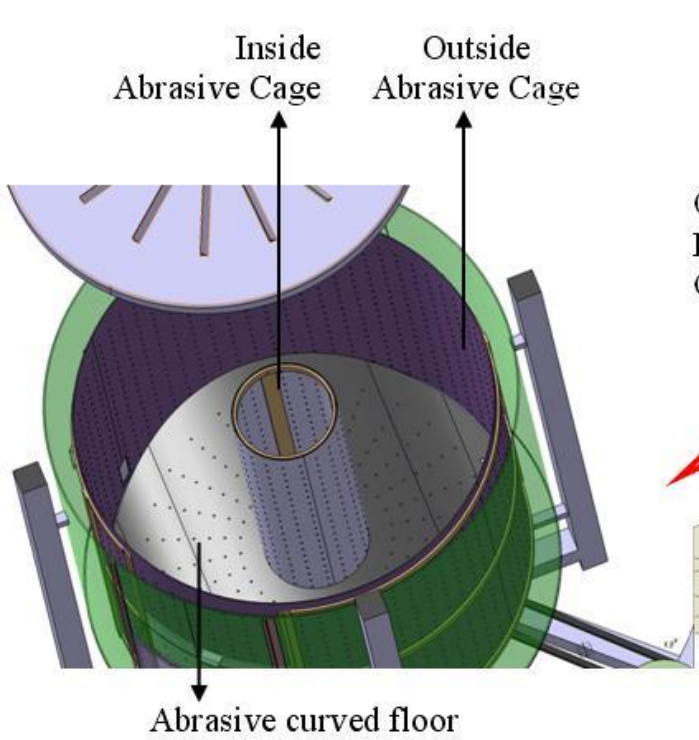

(a)

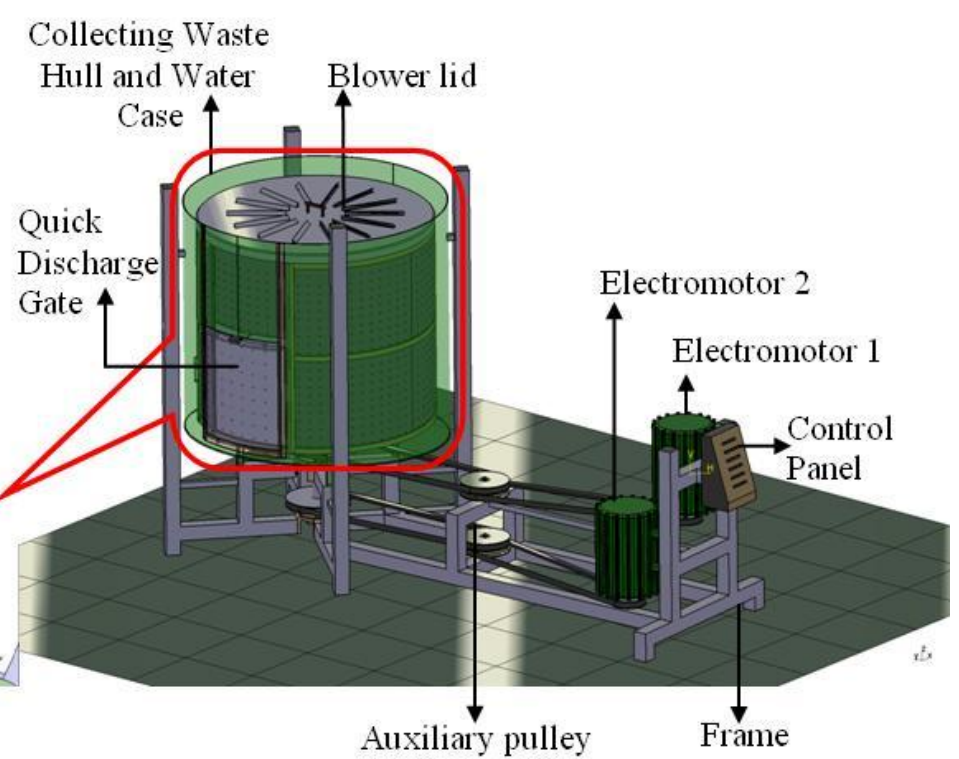

(b)

FIG. 1. General view of the proposed multipurpose machine: (a) process chamber of the machine, (b) different parts of the machine.

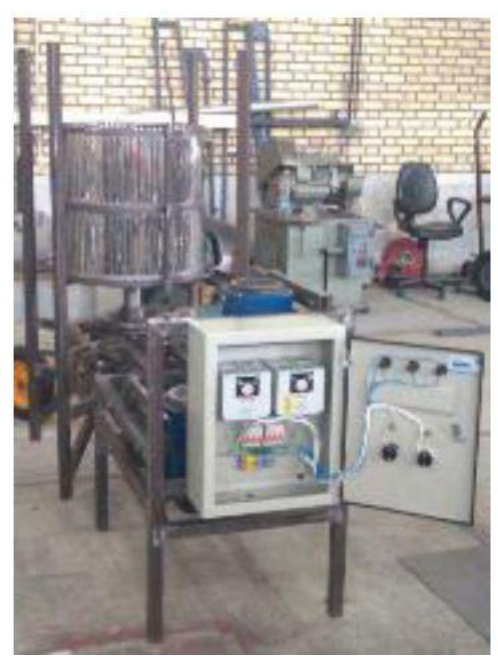

(a)

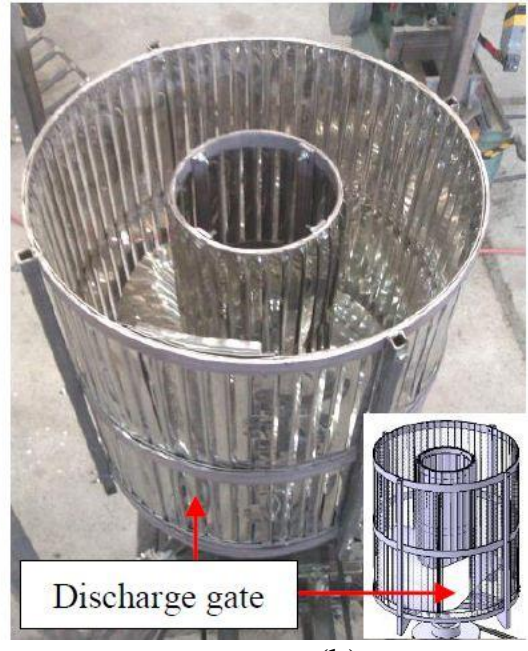

(b)

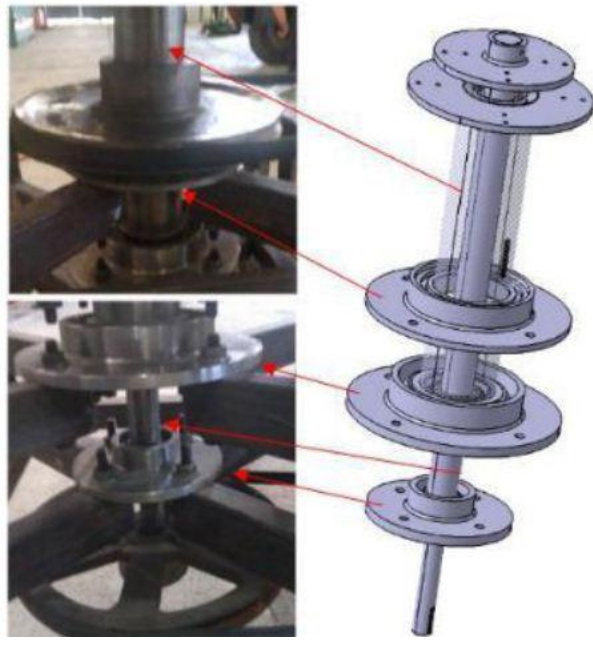

(c)

FIG. 2. Constructed laboratory sample of the multipurpose machine: (a) control panel installed on the machine contains two frequency inverters, (b) process chamber build by slotted walls, (c) the multi-drive coupling system that transmit power to OAC and IAC and curved bottom. 

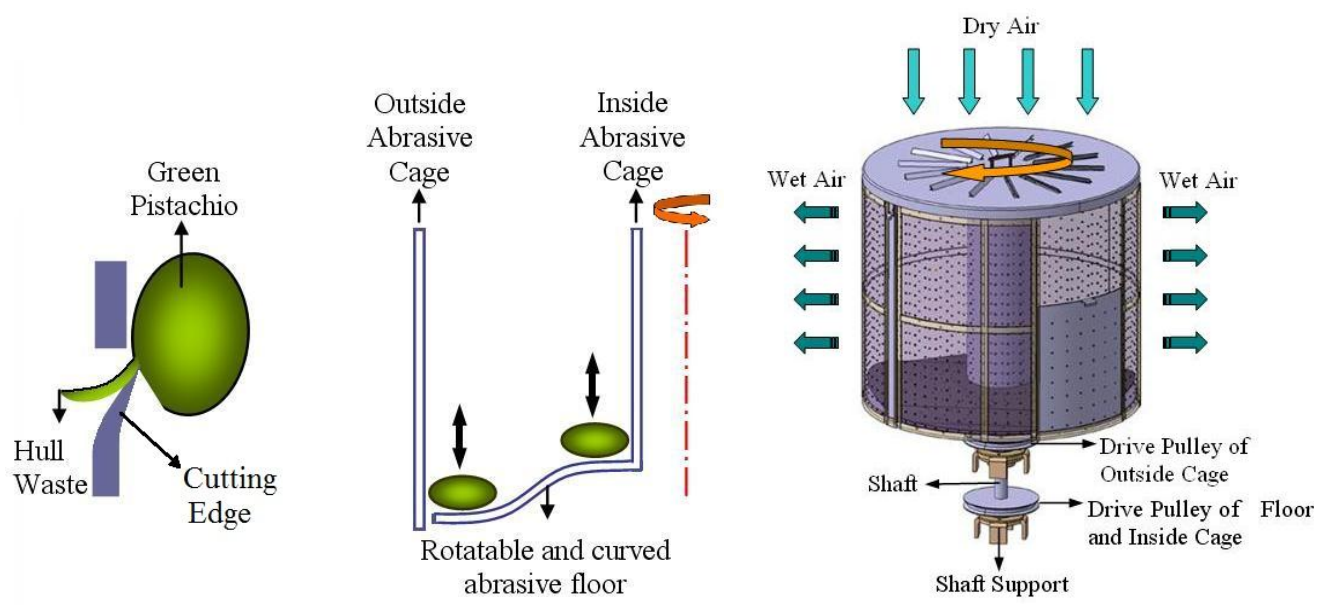

(a)

(b)

(c)

FIG. 3. Schematic view of the working principles of pistachio multipurpose machine: (a) hull removing of a nut, (b) hull removing principles, (c) airflow blowing through process chamber drains the washed nuts.

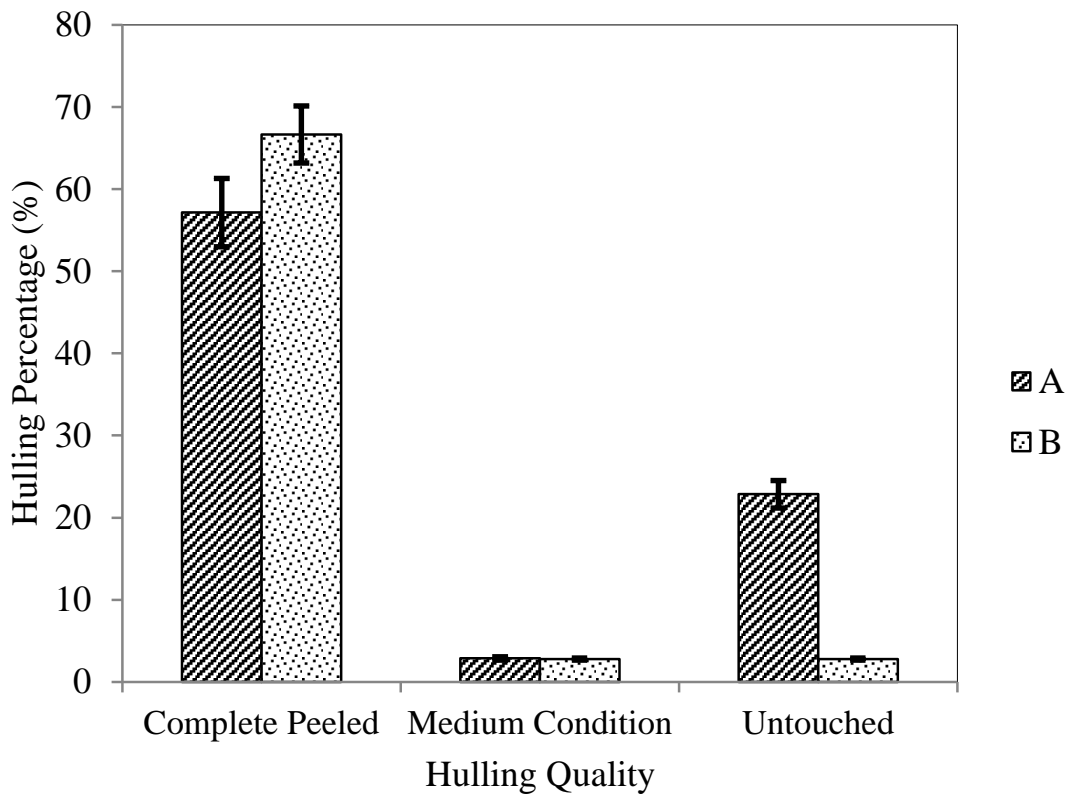

FIG. 4. Quality assortment of the output after hulling process of pistachio. 


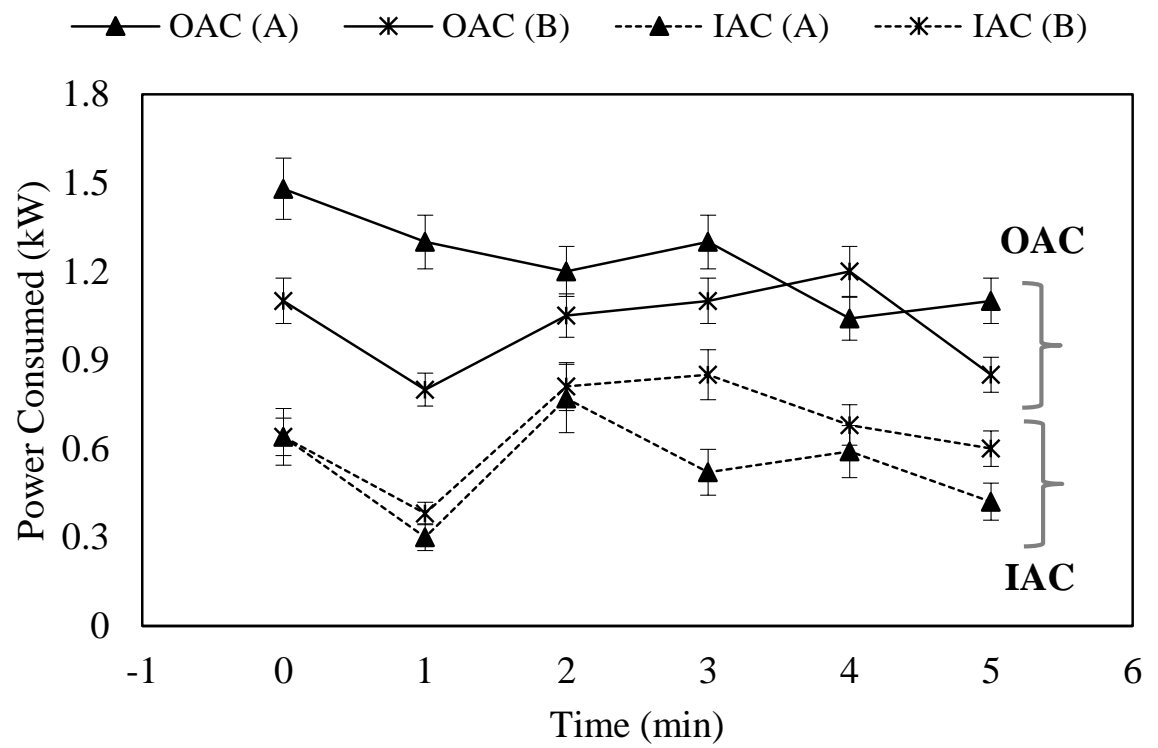

FIG. 5. Energy consumption of the process chamber in: (a) treatment A, and (b) treatment B.

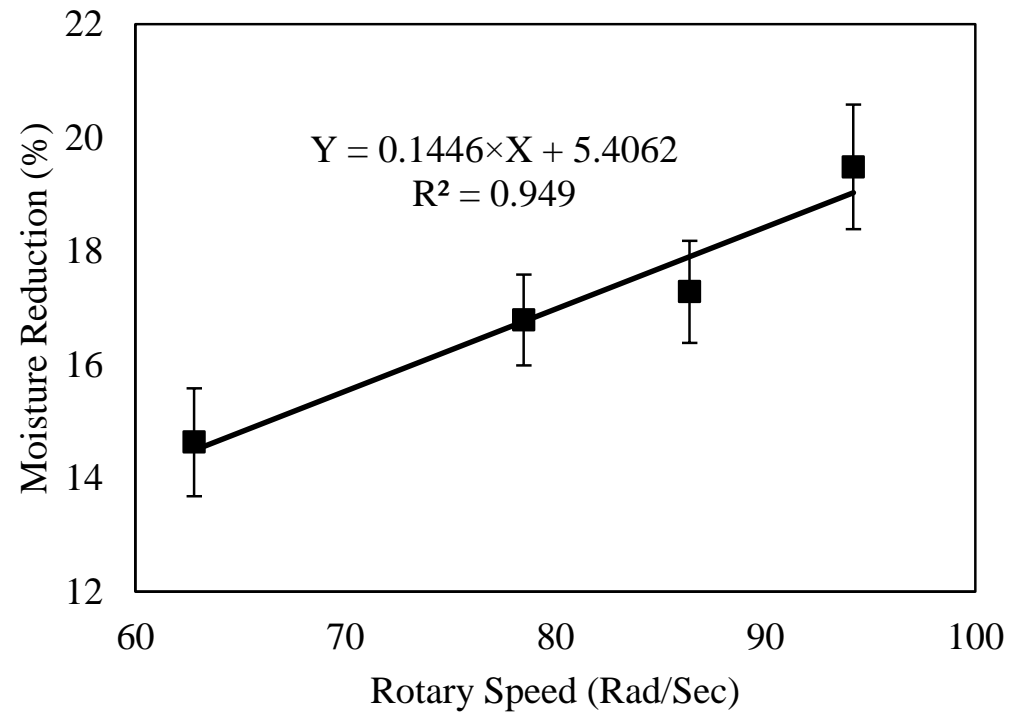

FIG. 6. Draining right after washing process decrease the whole moisture content. Error bars indicate variation of data around the average measured from five samples. 


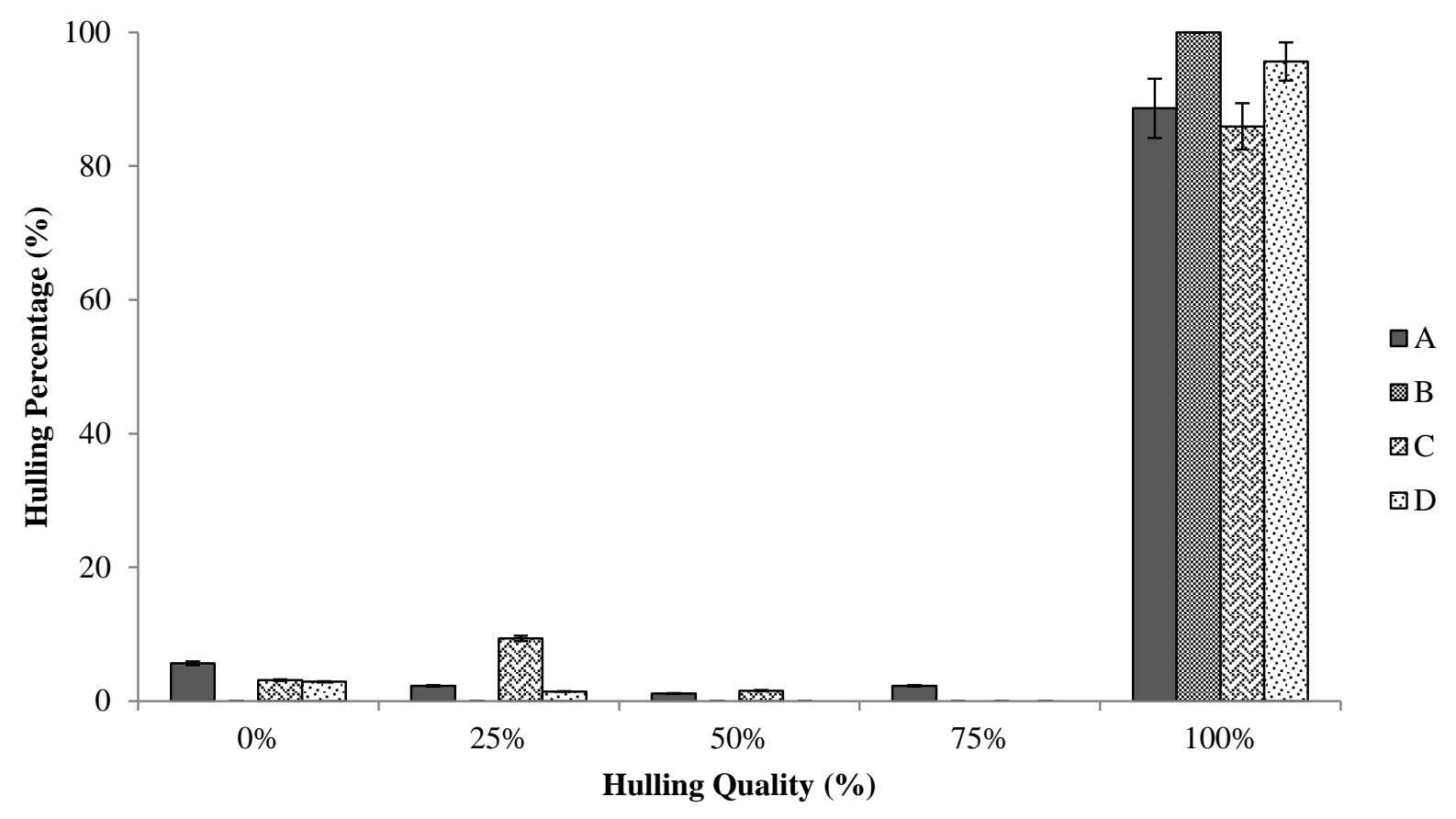

FIG. 7. Quality assortment of the output of the machine after hulling walnuts.

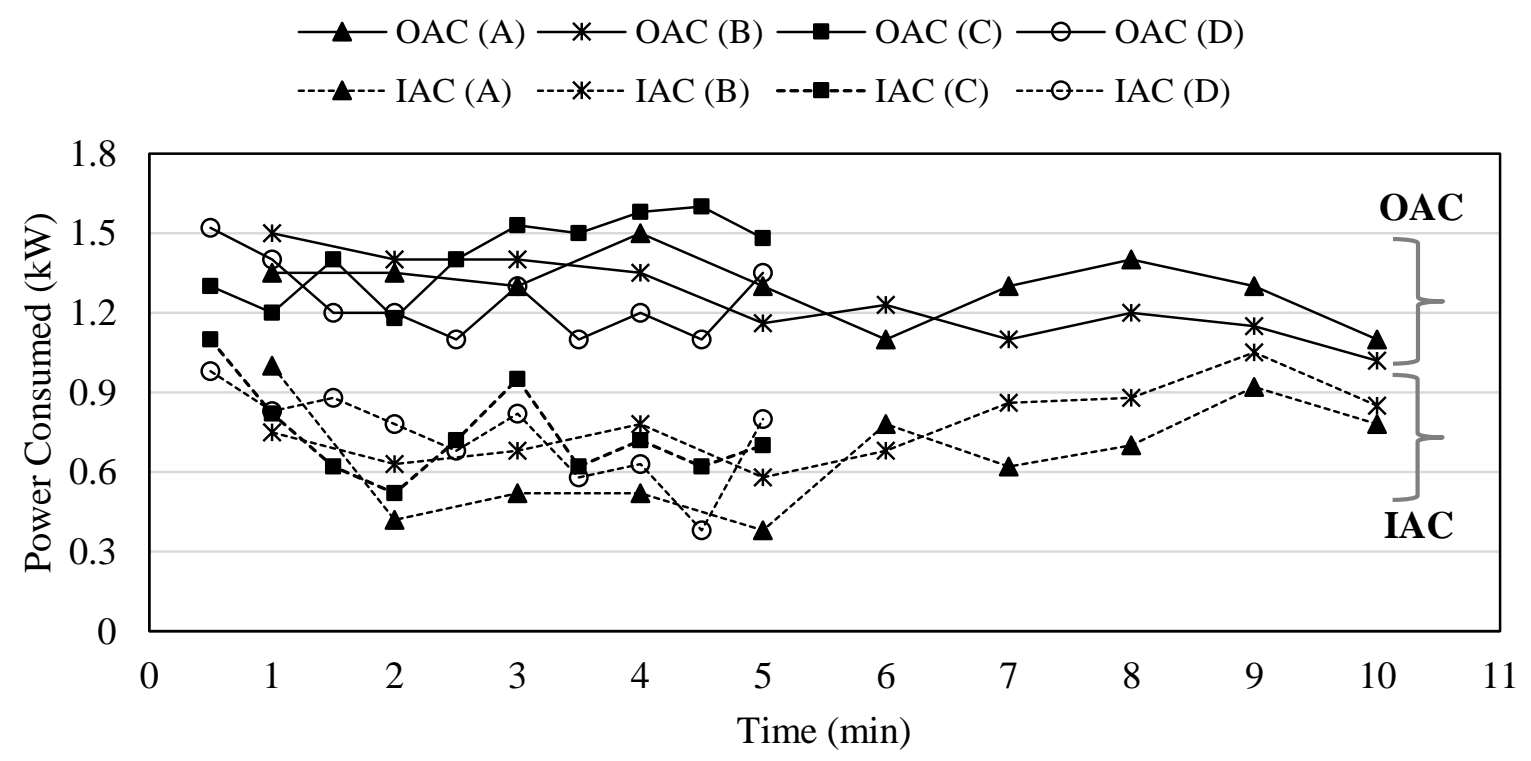

FIG. 8. Power consumption during hulling process of walnuts under treatments A, B, C and D. 
Table 1. Treatments for evaluating the hulling ability of the machine for pistachio nuts.

\begin{tabular}{|c|c|c|c|c|}
\hline Treatment & Nut's Weight $(\mathrm{kg})$ & Time $(\mathrm{min})$ & OAC rev $(\mathrm{rad} / \mathrm{s})$ & IAC rev $(\mathrm{rad} / \mathrm{s})$ \\
\hline $\mathrm{A}$ & $4(\times 4)$ & 5 & 2.5 & -3.75 \\
\hline $\mathrm{B}$ & $4(\times 4)$ & 5 & 3.75 & -2.5 \\
\hline
\end{tabular}

Table 2. Treatments for evaluating the hulling ability of the machine for walnuts.

\begin{tabular}{|c|c|c|c|c|}
\hline Treatment & Nut's Weight $(\mathrm{kg})$ & Time $(\mathrm{min})$ & OAC rev $(\mathrm{rad} / \mathrm{s})$ & IAC rev $(\mathrm{rad} / \mathrm{s})$ \\
\hline $\mathrm{A}$ & $4(\times 4)$ & 10 & 2.5 & -3.75 \\
\hline $\mathrm{B}$ & $4(\times 4)$ & 10 & 3.75 & -2.5 \\
\hline $\mathrm{C}$ & $4(\times 4)$ & 5 & 2.5 & -3.75 \\
\hline $\mathrm{D}$ & $4(\times 4)$ & 5 & 3.75 & -2.5 \\
\hline
\end{tabular}

Table 3.Analysis of the variance of parameters considered on hulling performance.

\begin{tabular}{|l|l|l|l|}
\hline Nut & Variation source & Df & Mean Square \\
\hline \multirow{3}{*}{ Pistachio } & Between Treatments & 3 & $165.407 * *$ \\
\cline { 2 - 4 } & Error & 12 & 7.743 \\
\hline \multirow{3}{*}{ Walnut } & Between Treatments & 1 & $183.170 * *$ \\
\cline { 2 - 4 } & Error & 6 & 12.856 \\
\hline & $* *$ Significance at 1\% level. \\
\hline
\end{tabular}

Table 4. Mean comparison of hulling performance (\%) in different treatments.

\begin{tabular}{|c|c|c|c|c|c|c|}
\hline \multirow{2}{*}{ Treatments } & \multicolumn{2}{|c|}{ Pistachio } & \multicolumn{4}{|c|}{ Walnut } \\
\hline & A & B & A & B & $\mathrm{C}$ & $\mathrm{D}$ \\
\hline \multirow[t]{2}{*}{ Performance } & $57.1 \mathrm{~b}$ & $66.67 \mathrm{a}$ & $88.64 \mathrm{c}$ & $100 \mathrm{a}$ & $85.94 \mathrm{c}$ & $95.65 \mathrm{~b}$ \\
\hline & \multicolumn{2}{|c|}{$\begin{array}{l}\text { The means with the same letter is not } \\
\text { significant at } 5 \% \text { level according to } \\
\text { Duncan's multiple ranges test. }\end{array}$} & \multicolumn{4}{|c|}{$\begin{array}{l}\text { The means with the same letter is not } \\
\text { significant at 5\% level according to } \\
\text { Duncan's multiple ranges test. }\end{array}$} \\
\hline
\end{tabular}

Table 5. Consuming power and standard deviation in treatments A and B

\begin{tabular}{|l|l|l|l|l|l|l|l|}
\hline \multirow{3}{*}{ Treatments: } & $\begin{array}{l}\text { Power used } \\
(\mathrm{kW}):\end{array}$ & \multirow{2}{*}{ STDV } & $\begin{array}{l}\text { Power used } \\
(\mathrm{kW}):\end{array}$ & \multirow{2}{*}{ STDV } & $\begin{array}{l}\text { Total power } \\
\text { used }(\mathrm{kW}):\end{array}$ & \multicolumn{2}{l|}{$\begin{array}{l}\text { Percent of total } \\
\text { power }(\%):\end{array}$} \\
\cline { 7 - 9 } & & OAC & IAC & & & OAC & IAC \\
\hline $\mathrm{A}^{*}$ & 1.237 & 0.159 & 0.54 & 0.166 & 1.777 & 69.61 & 30.39 \\
\hline $\mathrm{B}^{*}$ & 1.017 & 0.152 & 0.66 & 0.168 & 1.66 & 60.24 & 39.76 \\
\hline$*$
\end{tabular}


Table 6. Average of power consumed by OAC and IAC in

\begin{tabular}{|c|c|c|c|c|c|c|c|}
\hline \multirow[t]{2}{*}{ Treatments: } & \multirow{2}{*}{$\begin{array}{l}\text { Power } \\
\text { used }(\mathrm{kW}) \text { : } \\
\text { OAC }\end{array}$} & \multirow[t]{2}{*}{ STDV } & \multirow{2}{*}{$\begin{array}{l}\text { Power } \\
\text { used }(\mathrm{kW}) \text { : } \\
\text { IAC }\end{array}$} & \multirow[t]{2}{*}{ STDV } & \multirow{2}{*}{$\begin{array}{l}\text { Total power } \\
\text { used }(\mathrm{kW}) \text { : }\end{array}$} & \multicolumn{2}{|c|}{$\begin{array}{l}\text { Percent of total } \\
\text { power }(\%) \text { : }\end{array}$} \\
\hline & & & & & & OAC & IAC \\
\hline $\mathrm{A}^{*}$ & 1.300 & 0.122 & 0.664 & 0.208 & 1.964 & 66.19 & 33.81 \\
\hline $\mathrm{B}^{*}$ & 1.251 & 0.154 & 0.774 & 0.140 & 2.025 & 61.78 & 38.22 \\
\hline $\mathrm{C}^{* *}$ & 1.417 & 0.150 & 0.739 & 0.175 & 2.156 & 65.72 & 34.28 \\
\hline $\mathrm{D}^{* *}$ & 1.247 & 0.143 & 0.736 & 0.172 & 1.983 & 62.88 & 37.12 \\
\hline
\end{tabular}

